\title{
Algebraic Objects of MBFs and Recursive Computation of the Dedekind Number
}

\author{
Tkachenco V.G. ${ }^{1, *}$, Sinyavsky O.V. ${ }^{2}$ \\ ${ }^{1}$ Institute of Radio, Television, Electronics, Odessa National Academy of Telecommunications, Ukraine \\ ${ }^{2}$ Department of Fundamental Sciences, Odessa Military Academy, Ukraine
}

Copyright $(2017$ by authors, all rights reserved. Authors agree that this article remains permanently open access under the terms of the Creative Commons Attribution License 4.0 International License

\begin{abstract}
In this article the whole set of n-1 rank Monotone Boolean Functions (MBFs) is divided into equivalence classes. It shows how the Dedekind number $\mathrm{D}(\mathrm{n})$ can be calculated by using this partition. Five formulas were found to calculate this number as well as the algebraic properties of MBF blocks.
\end{abstract}

Keywords Monotone Boolean Functions, Free Distributive Lattice, Dedekind Number, Algebraic Structures

\section{Introduction}

In $1897 \mathrm{R}$. Dedekind found $\psi(4)$ number of elements for a free distributive lattice with four generators, which he published in his article [1]. In algebra parlance the Dedekind number $D(n)=\psi(n)+2$ is the number of the Monotone Boolean functions (MBFs) dependent on $n$ number of $x_{1}, \ldots$, $x_{n}$ variables. $D(0)-D(4)$ were found by Dedekind. $D(5)$ was calculated by Church [2] in 1940, D(6) - by Ward [3] in 1946, $D(7)$ - by Church [4] in 1965 and $D(8)$ - by Wiedemann [5] in 1991. The inequivalent MBFs were conducted until the seventh rank in [6]. As it turned out, this problem is quite difficult and not manageable under the traditional method of generating functions. There is no a simple closed formula for finding the Dedekind number [7].

However, the recursive method to find the Dedekind number $D(n)$ by dividing all the elements of the free distributive lattice of $n-1$ rank MBFs into equivalence classes is not reported. In some cases, this method reduces the search of MBFs due to it easier to consider the only $n-1 \mathrm{MBFs}$ (which are significantly less in number) instead of considering all $n$ - 1 rank MBFs.

The aim of this paper is to develop a new method of recursive calculation of the Dedekind number $D(n)$ based on the $n-1$ MBFs equivalence classes partition and to study the algebraic properties of the MBF blocks.

\section{Results}

Let's consider the process of recursion of an $n$-rank MBF from an $n-1$ rank MBF.

Let's map $P:\left(f_{i}(n-1), f_{j}(n-1)\right) \rightarrow f(n)$ of all the $\mathrm{n}-1$ rank MBF pairs on an n-rank MBF as follows: $f_{k}(n)=f_{i}(n-1) x_{n} \vee f_{j}(n-1)$. Let $f_{i}(n-1)$ be the left or upper part of $f_{k}(n)$, and the rest of $f_{j}(n-1)-$ its right or lower part.

Let's call a binary notation of a function of its value on all ordered sets of variables. The binary bits will be numbered from right to left from 0 to $2^{n}-1$, where $n$ is a rank of MBF. Let's select unity in bit $j$ of the MBF binary notation. Let's call subordinated all the MBF's unities in bits $i>j$ where the MBF stops being monotone if any of the bits changes in 0 . Define the dependent units for a given unit in the $j$-bit. This is the unit itself in the $\mathrm{j}$-bit, as well as the units in the bits $i \leq j$ that need to be zeroed out when the unit is zeroed in the $j$-bit in order for the MBF to remain monotonic.

Example 1. The binary notation of 3-rank MBF is 1111 1111, where the bits of this representation are numbered from right to left from 0 to 7 . Unity from bit 0 is self-dependent and all other unities are subordinated. There no subordinates for unity from bit 7 but all of them are dependent. Unities in bits $0,2,4$ and 6 are dependent for unity from bit 6 , and unity in bit 7 is subordinated. Unities in bits 0 and 4 are dependent for unity from bit 4 , and unities from bits 6 and 7 are subordinates.

The binary notation of $f_{k}(n)$ consists of two equal parts: $2^{n-1}$ which represents the left part (where $x_{n}=1$ ) and the right part (where $x_{n}=0$ ). Each part represents an $n$-1 rank MBF, at that $f_{i}(n-1) \geq f_{j}(n-1)$. This is the "greater-than-or-equal-to" for all the elements of the free distributive lattice of the $n-1$ rank MBF.

If the upper part of $f_{i}(n-1)$ is replaced with $f_{i}(n-1) \vee f_{j}(n-1)$, the upper part of the binary notation 
does not change, because $f_{i}(n-1) \geq f_{j}(n-1)$.

It is known that conjunction $s$ absorbs conjunction $t$ if all its variables are part of conjunction $t$. Let's say that MBF $f$ absorbs MBF $g$, if each conjunction $g$ is absorbed by one of conjunction $f$. It is equivalent for the binary notation that $f \geq g$, i.e. one function is more than the other. It follows from this inequality that $f=f \vee g$, and $g=f \wedge g$.

Let's designate monotone a pair of functions, where $f_{i}(n-1) \geq f_{j}(n-1)$. Indeed, the concatenation of binary notations for such pairs is the binary notation of an $n$-rank MBF by the definition of monotonicity.

For example, this pair of functions: disjunction functions $f_{i}(n-1) \vee f_{j}(n-1)$ and $f_{j}(n-1)$ is always monotone, i.e. the upper part of the MBF is disjunction of $f_{i}(n-1) \vee f_{j}(n-1)$.

Assume that all the set of pairs of an $n-1$ rank MBF are divide into equivalence classes. The two pairs fit the same equivalence class, if $P$ maps them onto the same $n$-rank MBF.

Example 2. Let's take a pair of 4-rank functions $f_{i}(4)=x_{2} x_{3} \vee x_{2} x_{4} \vee x_{3} x_{4}$ and $f_{j}(4)=x_{1} x_{2} \vee x_{1} x_{3} \vee x_{1} x_{4}$, along with another one pair $f_{r}(4)=x_{2} x_{3} \vee x_{2} x_{4} \vee x_{3} x_{4} \vee x_{1} x_{2} \vee x_{1} x_{3} \vee x_{1} x_{4}$ and $f_{s}(4)=x_{1} x_{2} \vee x_{1} x_{3} \vee x_{1} x_{4}$. These two pairs of functions are mapped by $P$ onto function $f(5)=x_{1} x_{2} \vee x_{1} x_{3} \vee x_{1} x_{4} \vee x_{2} x_{3} x_{5} \vee x_{2} x_{4} x_{5} \vee x_{3} x_{4} x_{5}$. I.e. $P\left(f_{i}, f_{j}\right)=P\left(f_{r}, f_{s}\right)=f(5)$.

Let's consider the factor set of function pairs against mapping $P$ and show what form each equivalence class of the factor set takes.

It is true that:

Lemma 1. For any pair of $\left(f_{i}(n-1), f_{j}(n-1)\right)$ disjunction $f_{i}(n-1) \vee f_{j}(n-1)$ and the second function $f_{j}(n-1)$ are constant within the whole equivalence class.

Proof.

$$
\begin{aligned}
f_{k}(n) & =f_{i}(n-1) x_{n} \vee f_{j}(n-1)= \\
& =f_{i}(n-1) x_{n} \vee f_{j}(n-1)\left(x_{n} \vee 1\right)= \\
& =f_{i}(n-1) x_{n} \vee f_{j}(n-1) x_{n} \vee f_{j}(n-1)= \\
& =\left(f_{i}(n-1) \vee f_{j}(n-1)\right) x_{n} \vee f_{j}(n-1)
\end{aligned}
$$

is correct for any pair of $f_{i}(n-1)$ and $f_{j}(n-1)$ from the same class.

It follows that the disjunction of any pair $f_{i}(n-1) \vee f_{j}(n-1)$ of one equivalence class is constant for the entire equivalence class. Otherwise $f_{k}(n)$ would be different and have another equivalence class.

Further, if the second function $f_{j}(n-1)$ is changed, the binary notation will be changed too, and the pair will be mapped onto another function, i.e. we will get another equivalence class. Therefore, the second function $f_{j}(n-1)$ is always one and the same for all pairs of functions within each equivalence class. The lemma is proved.

Another equivalence class definition can be given based on this lemma and the definition of mapping $P$ :

Definition. Function pairs $f_{i}(n-1)$ and $f_{j}(n-1)$ are from the same equivalence class if they form one and the same disjunction $f_{i}(n-1) \vee f_{j}(n-1)$ and the second function $f_{j}(n-1)$ does not change.

Lemma 2. Let's consider the equivalence class, to which pair $f_{i}(n-1)$ and $f_{j}(n-1)$ belongs. Then there is a single pair of monotone functions: disjunction $f_{i}(n-1) \vee f_{j}(n-1)$ and $f_{j}(n-1)$ for any pair of $\left(f_{i}(n-1), f_{j}(n-1)\right)$ within each equivalence class.

Proof. Let's consider mapping P, $f_{i}(n-1) x_{n} \vee f_{j}(n-1)$, when $x_{n}=1$ and when $x_{n}=0$

When $x_{n}=1$

$f_{i}(n-1) x_{n} \vee f_{j}(n-1)=f_{i}(n-1) 1 \vee f_{j}(n-1)=f_{i}(n-1) \vee f_{j}(n-1)$

When $x_{n}=0 \quad x_{n}=1$

$f_{i}(n-1) x_{n} \vee f_{j}(n-1)=f_{i}(n-1) 0 \vee f_{j}(n-1)=f_{j}(n-1)$

It follows that $f_{k}(n)=f_{i}(n-1) x_{n} \vee f_{j}(n-1)$ represents a concatenation of two binary functions: $f_{i}(n-1) \vee f_{j}(n-1)$ is the first and $f_{j}(n-1)$ is the second. The pair is monotone because $f_{i}(n-1) \vee f_{j}(n-1) \geq f_{j}(n-1)$. Further, taking into account lemma 1, that disjunction $f_{i}(n-1) \vee f_{j}(n-1)$ is constant and the second function $f_{j}(n-1)$ is always one and the same for all pairs of functions, it follows that there can only be the only one monotone pair within a certain equivalence class. The lemma is proved.

Example 3. Let $f(5)=f_{1}(4) x_{5} \vee f_{2}(4)$. If $f_{1}(4)=x_{2} x_{3} \vee x_{2} x_{4} \vee x_{3} x_{4} \vee x_{1} x_{2} \vee x_{1} x_{3} \vee x_{1} x_{4} \quad$ and $f_{2}(4)=x_{1} x_{2} \vee x_{1} x_{3} \vee x_{1} x_{4}$, the pair will be monotone, and if $f_{1}(4)=x_{2} x_{3} \vee x_{2} x_{4} \vee x_{3} x_{4}$ and $f_{2}(4)=x_{1} x_{2} \vee x_{1} x_{3} \vee x_{1} x_{4}$, the pair will be nonmonotone. Here the monotony is broken by the following setups:

Table 1. Nonmonotonicity $f(5)=f_{1}(4) x_{5} \vee f_{2}(4)$

\begin{tabular}{|c|c|c|c|c|c|c|c|c|}
\hline$x_{5}$ & $x_{4}$ & $x_{3}$ & $x_{2}$ & $x_{1}$ & $f_{1}$ & $f_{2}$ & $f_{1} x_{5}$ & $f_{1} x_{5} \vee f_{2}$ \\
\hline 1 & 0 & 0 & 1 & 1 & 0 & 1 & 0 & 1 \\
\hline 1 & 0 & 1 & 0 & 1 & 0 & 1 & 0 & 1 \\
\hline 1 & 1 & 0 & 0 & 1 & 0 & 1 & 0 & 1 \\
\hline
\end{tabular}

The pair of functions $f_{i}(n-1)$ and $f_{j}(n-1)$ is 
monotone. So the first function is disjunction $f_{i}(n-1) \vee f_{j}(n-1)$ and the second is constant. Therefore, all the functions of the equivalence class can be got by modifying the first function. Let's write the binary notation of the first function. Other functions can be got from this one by only substituting some unities for 0 . It cannot be done vice versa due to the first function is equal to $f_{i}(n-1) \vee f_{j}(n-1)$ and it is a maximal one. Next, only those unities can be substituted for 0 that have dependent in both the first and the second MBFs in order not to change the equivalence class and not to break the monotony of function $f_{i}(n-1)$. Otherwise disjunction $f_{i}(n-1) \vee f_{j}(n-1)$ will be broken and therefore, it will be another equivalence class.

Example 4 . $f_{i}(3)=x_{2} x_{3}=11000000$, $f_{j}(3)=x_{1} \vee x_{2} \vee x_{3}=11111110$. Then $f_{i}(n-1) \vee f_{j}(n-1)=11111110$. So we will get 19 functions by substituting unities for 0 in $f_{i}(n-1) \vee f_{j}(n-1)$ in accordance with the above rule: $f_{1}(3)=x_{1}=10101010, \quad f_{2}(3)=x_{2}=11001100, \quad \ldots$, $f_{17}(3)=x_{2} x_{3}=11000000, f_{18}(3)=x_{1} x_{2} x_{3}=10000000$, $f_{19}(3)=00000000$. I.e. this equivalence class consists of 19 pairs of functions.

Every other function $f_{j}(n-1)$ sets partition for the set of all $n-1$ rank MBFs. All the MBFs, which form pairs with the second function and are within the same equivalence class, will belong to the same partition class. Let's symbolize this partition $Q_{j}$.

Lemma 3. Each class of partition $Q_{j}$ represents an interval on the distributive lattice of all $n$-1rank MBFs.

Proof. Let the second function $f_{j}(n-1)$ is constant on the set of definitions for mapping $P$. Then it sets partition $Q_{j}$ on the set of $n-1$ rank MBFs, and the whole equivalence class will be symbolized $T$. If we take any function from one class of partition $Q_{j}$ as the first function of pairs $f_{i}(n-1)$ and $f_{j}(n-1)$, then $P$ will map these pairs onto one function.

Let's consider any $f_{1}, f_{2} \in T$. Then $P$ maps pairs $f_{1} \vee f_{j}$ and $f_{2} \vee f_{j}$ onto one and the same function. We are going to prove that $f_{1} \vee f_{2} \in T$ and $f_{1} \wedge f_{2} \in T$. It follows from lemma 1 that $f_{1} \vee f_{j}=f_{2} \vee f_{j}$. Besides $\left(f_{1} \vee f_{2}\right) \vee f_{j}=\left(f_{1} \vee f_{j}\right) \vee\left(f_{2} \vee f_{j}\right)$, i.e. $f_{1} \vee f_{2} \in T$

If $f_{j} \in Q_{j}$, then $f_{1} \vee f_{j}$ and $f_{2} \vee f_{j}$ are equal to the constant disjunction for this class of equivalence $f_{i} \vee f_{j}$ (see. lemma 1). As the lower part of function $f_{j}$ is one and the same in the equivalence class, then $f_{i} \vee f_{j}=\left(f_{1} \vee f_{j}\right) \wedge\left(f_{2} \vee f_{j}\right)=f_{1} f_{2} \vee f_{j}$, i.e. $f_{1} \wedge f_{2} \in T$.

Therefore, $T$ is sublattice of the lattice for all $n-1$ rank MBFs. As far as this sublattice is finite, it has a minimum and a maximum element. $f_{i} \vee f_{j}$ is the maximum element of this sublattice. Let some $f_{m}$ is the minimum element of sublattice $T$ and some $f_{3}$ has such value that $f_{m} \leq f_{3} \leq f_{i} \vee f_{j}$. As far as $f_{m} \vee f_{j}=\left(f_{i} \vee f_{j}\right) \vee f_{j}$ is true for class $T$, then $f_{3} \vee f_{j}=f_{i} \vee f_{j}$. Therefore $f_{3} \in T$ and this sublattice is an interval. The lemma is proved.

Ideal $I_{i}$, generated by element $f_{i}$ of its distributive lattice, represents all the MBFs, which are less than or equal to $f_{i}$. Respectively, co-ideal $C_{i}$, generated by element $f_{i}$ of its distributive lattice, represents all the MBFs, which are greater than or equal to $f_{i}$. If $f_{a}$ and $f_{b}$ are the minimum and the maximum element of this interval, the interval is symbolized $\left[f_{a}, f_{b}\right]$. This interval represents the intersection of the $f_{b}$ maximum ideal and the $f_{a}$ minimum co-ideal.

Using the above determined order and that the second function is constant in its equivalence class, the minimum function $f_{m}$ in the class is that one which has no common conjunctions with the second function. There can be the only one function, because it is a sublattice of a distributive lattice. In other words the minimum function in an equivalence class can be either a zero function, or that one which has no conjunctions absorbed by the second MBF.

There is an equivalence class for any rank where $f_{j}(n-1)=1$ and $f_{i}(n-1)$ is any function of $n-1$ rank. The number of pairs in this class is equal to the number of $n-1$ rank MBFs. It is true for any class that it is greater than the number of $n-1$ rank MBFs by 1 if $f_{j}(n-1)=x_{1} \vee x_{2} \vee \ldots \vee x_{n-1}$, and $f_{i}(n-1)$ is any function but a unit one in this class.

Any function of $n$-rank can be represented by an equivalence class.

Example 5. Let's consider a function with the maximum number of conjunctions: $f(5)=x_{1} x_{2} x_{3} \vee x_{1} x_{2} x_{4} \vee x_{1} x_{2} x_{5} \vee$ $x_{1} x_{3} x_{4} \vee x_{1} x_{3} x_{5} \vee x_{1} x_{4} x_{5} \vee x_{2} x_{3} x_{4} \vee x_{2} x_{3} x_{5} \vee x_{2} x_{4} x_{5} \vee x_{3} x_{4} x_{5}$ This function can be represented as $f(5)=f_{1}(4) x_{5} \vee f_{2}(4)$, where $f_{1}(4)=x_{1} x_{2} \vee x_{1} x_{3} \vee x_{1} x_{4} \vee x_{2} x_{3} \vee x_{2} x_{4} \vee x_{3} x_{4} \quad$ and $f_{2}(4)=x_{1} x_{2} x_{3} \vee x_{1} x_{2} x_{4} \vee x_{1} x_{3} x_{4} \vee x_{2} x_{3} x_{4}$. The binary notation of these MBFs is $f_{1}(4)=1111111011101000$, $f_{2}(4)=1110100010000000$. It is obvious, that $f_{1}(4)>f_{2}(4)$, conjunctions of $f_{1}(4)$ absorb conjunctions of $f_{2}(4)$. In accordance with the rule of replacing unities (after example 3), only those unities can be deleted from $f_{1}(4)$, which dependents are both in the first and the second functions. But there are no unities to be deleted in $f_{1}(4)$ in accordance with this rule. For example, unity of bit 7 cannot be removed from $f_{1}(4)$ because its dependents are in bits 3 , 5 and 6 , which are not in $f_{2}(4)$. Therefore, in this case the 
equivalence class consists only of a single pair.

Lemma 4. The Dedekind number $D(n)$ is equal to the sum of the power co-ideals of the distribution lattice of all $n-1$ rank MBFs.

Proof. It follows from lemma 2, that there is a single monotone pair within any equivalence class. On the other hand some equivalence class contains each monotone pair. Consequently, the number of equivalence classes is equal to the number of monotone pairs. Since the monotone pairs are the range of values for mapping $P$, then the Dedekind number $D(n)$ is equal to the total number of equivalence classes. When considering a set of pairs from the constant second function, the only first functions should be taken which are greater than the second function $f_{j}(n-1)$ in order the pair remains monotone. Then $K_{j}$, which represents the number of classes in partition $Q_{j}$, is equal to the number of functions $f_{i}(n-1) \geq f_{j}(n-1)$. By definition this is power co-ideal $C_{j}$, generated by $f_{j}(n-1)$. The lemma is proved.

Several formulas can be obtained to calculate the Dedekind number based on the above:

1. $D(n)=\sum_{f_{j} \in M(n-1)} K_{j}$, where $K_{j}$ is the number of classes in partition $Q_{j}, M(n-1)$ is the set of all $n-1$ rank MBFs.

2. Another one formula to calculate the Dedekind number $D(n)=\sum_{f_{j}}\left|C_{j}\right|$ results from lemma 4 , where $\left|C_{j}\right|$ is power co-ideal $C_{j}$, generated by $f_{j}(n-1)$.

3. $D(n)=\sum_{f_{j}}\left|I_{j}\right|$ can be obtained from the symmetry of the distributive lattice of n-1 rank MBFs, where $\left|I_{j}\right|$ is power ideal $I_{j}$, generated by $f_{j}(n-1)$

4. The two previous formulas can be written as follows in order to simplify calculations: $D(n)=\sum_{f^{*}{ }_{j}}\left|C_{j}\right| \cdot k_{j}$ or $D(n)=\sum_{f^{*}{ }_{j}}\left|I_{j}\right| \cdot k_{j}$, where $k_{j}$ is the number of isomorphic functions to $f_{j}(n-1)$, and the summation is only on nonisomorphic $f^{*}$.

Example 6. There are $20 \mathrm{MBFs}$ of 3-rank. $f_{0}(3)$ is identically equal to $0 ; f_{1}(3)$ is identically equal to $1 ; f_{2}(3)=$ $x_{1} x_{2} x_{3} ; f_{3}(3)=x_{1} \vee x_{2} \vee x_{3} ; f_{4}(3)=x_{1} x_{2} \vee x_{1} x_{3} \vee x_{2} x_{3} ; f_{5}(3)=x_{1}$; $f_{6}(3)=x_{2} x_{3} ; f_{7}(3)=x_{2} \vee x_{3} ; f_{8}(3)=x_{1} x_{2} \vee x_{1} x_{3} ; f_{9}(3)=x_{1} \vee x_{2} x_{3}$; $f_{10}(3)=x_{2} ; f_{11}(3)=x_{1} x_{3} ; f_{12}(3)=x_{1} \vee x_{3} ; f_{13}(3)=x_{1} x_{2} \vee x_{2} x_{3}$; $f_{14}(3)=x_{2} \vee x_{1} x_{3} ; f_{15}(3)=x_{3} ; f_{16}(3)=x_{1} x_{2} ; f_{17}(3)=x_{1} \vee x_{2} ; f_{18}(3)$ $=x_{1} x_{3} \vee x_{2} x_{3}$ и $f_{19}(3)=x_{3} \vee x_{1} x_{2}$. The distributive lattice of a 3-rank MBF (Fig.1):

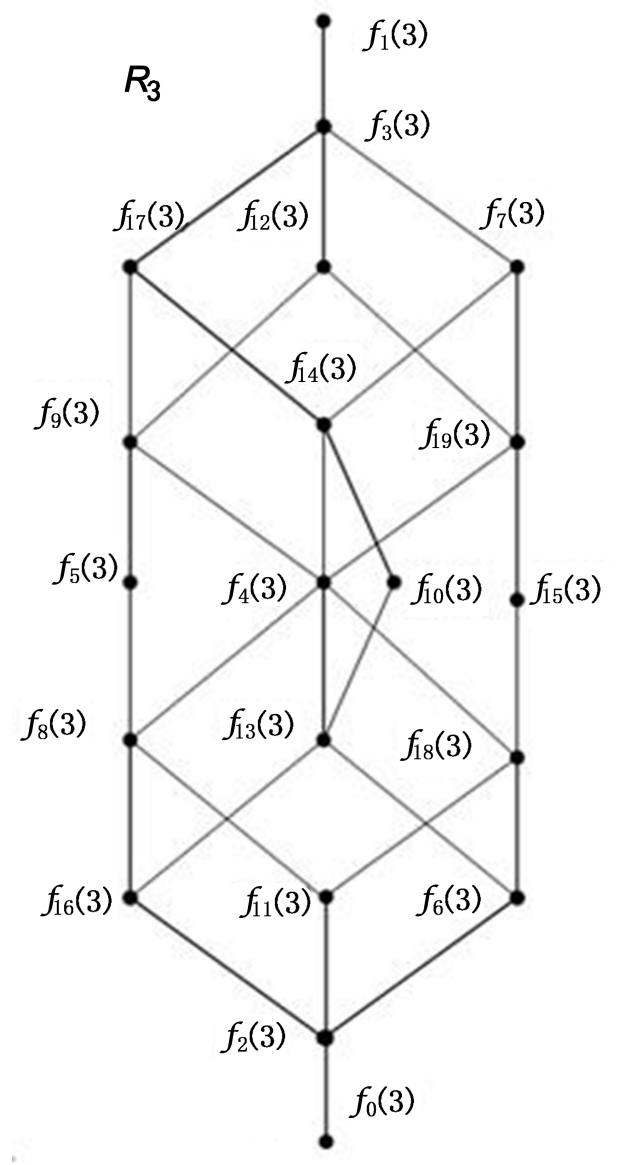

Figure 1. The distributive lattice of a 3-rank MBF We will get

$$
D(4)=20+1+19+2+9+6+14+3+11+5+6+14+3+11+5+6+14+3+11+5=168
$$


if we summarize all the 20 power co-ideals

But, since the power co-ideal is the same for isomorphic functions, the Dedekind number can be computed by summing up the products of power co-ideals and numbers of nonisomorphic MBFs. There are 10 pairwise nonisomorphic MBFs in the lattice of 3-rank MBFs $-f_{0}(3), f_{1}(3), f_{2}(3), f_{3}(3)$, $f_{4}(3), f_{6}(3), f_{8}(3), f_{5}(3), f_{9}(3), f_{7}(3)$. Moreover, the first 5 of them are isomorphic, and the other 5 have 2 additional isomorphic functions. It follows that

$$
D(4)=20+1+19+2+9+14 \cdot 3+11 \cdot 3+6 \cdot 3+5 \cdot 3+3 \cdot 3=168 \text {. }
$$

Let's take a closer look at the algebraic structure of the MBF blocks.

There are three unary operations in the set of MBFs of any rank [8]. Let's symbolize duality by $a$, disjunctive complement by $b$ and conjunctive complement by $c$. To obtain a disjunctive complement $\overline{f_{i}}(n)$ from an $i \operatorname{MBF} f_{i}(n)$ in the minimum disjunctive form each conjunction of $m$ variables must be replaced with the conjunction of all $n-m$ variables, which are not included in the initial conjunction. To obtain a conjunctive complement $\underline{f_{i}}(n)$ from an $i \mathrm{MBF}$ $f_{i}(n)$ in the minimum conjunctive form each disjunction of $m$ variables must be replaced with the disjunction of all $n-m$ variables, which are not included in the initial disjunction. To obtain a dual MBF $f_{i}^{-1}(n)$ from an $i \operatorname{MBF} f_{i}(n)$ in the minimum disjunctive form all the operations of conjunction must be replaced with the operations of disjunction and vice versa simultaneously. At this the dual $\operatorname{MBF} f_{i}^{-1}(n)$ is in the minimum conjunctive form. To obtain a dual $\operatorname{MBF} f_{i}^{-1}(n)$ in the minimum disjunctive form the brackets must be expanded and the similar terms must be combined in the received minimum conjunctive form.

Definition. A block of MBFs is a set of MBFs, which includes three operations - duality, disjunctive and conjunctive complement - so that any MBF of the block can be obtained from any MBF of the same block using a certain sequence of these three operations.

For example, there is the only one block for ranks 0 and 1 , which consists of two functions in the first case and of three functions in the second one (Figure 2).
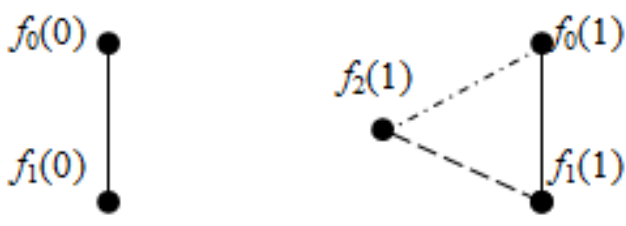

Figure 2. The MBF blocks of 0 and 1 ranks.

Here the solid line symbolize the operation of duality, the dashed line - the operation of disjunctive complement and the dot-and-dash line - the operation of conjunctive complement, $f_{0}(n)$ is a zero MBF (equal to 0 for all the sets of variables), $f_{1}(n)$ is a unit MBF (equal to 1 for all the sets of variables), $f_{2}(1)=x_{1}$
The MBF block that contains both $f_{0}(n)$ and $f_{1}(n)$ will be the base block.

Lemma 5. The conjunctive complement can be obtained for $f(n)$, by deriving dual functions from this function. The same is true for the disjunctive complement.

Proof. It is well known that dual function $g(n)$ can be obtained by replacing the operations of conjunction with the operations of disjunction in original $f(n)$ adhering to the precedency. And vice versa if the same replacement is held in the dual function, the original function will be obtained written in CNF. It follows that the conjunctive complement of $f(n)$ and the disjunctive complement of $g(n)$ will be dual to each other. The lemma is proved.

\section{Conclusions}

A block of MBFs may be defined as a set of MBFs, which includes three operations - duality, disjunctive and conjunctive complement - so that any MBF of the block can be obtained from any MBF of the same block using a certain sequence of two operations $a$ and $b$ only.

Thus, a block of function can be obtained from function $f$ by applying in turn the operation of disjunction first and then the operation of duality for function $f$. And each function can belong to the only one block.

Let's introduce some concepts. A block power is the number of MBFs, which the block includes. Two blocks are similar if they have the same power and are indistinguishable disregarding their MBFs. Two blocks are isomorphic, if any MBF of one block can be obtained from some MBF of another block by some variable substitution. Isomorphic blocks are similar by definition.

All MBF blocks can be divided into 4 types. It follows from lemma 5 that all MBF can be correlated using a sequence of two operations - the operation of duality and the operation of disjunctive complement. The sequence of these two operations can be open or cyclic. There are three types of blocks in the first case. The first type is referred to a block which has two disjunctive self-complementary MBFs at the ends of the open sequence. The second type is referred to a block which has a self-dual MBF at one end of the sequence and a disjunctive self-complementary MBF at the other. The third type is referred to a block which has two self-dual MBFs at the ends of the open sequence. There are no self-dual and disjunctive self-complementary MBFs in the second case. The fourth type is cyclic. There are 4 types of MBF blocks in total:

Containing two disjunctive self-complementary MBFs;

Containing one disjunctive self-complementary MBF and one self-dual MBF;

Containing two self-dual MBFs;

Cyclic blocks. All the functions of a block are correlated by the three operations. 
Another (the fifth) formula was developed to calculate the Dedekind number in [9] using the types of blocks. The Dedekind number equals to the number of MBFs in the isomorphic blocks. $D(n)=\sum C_{i 1} \cdot C_{i 2} \cdot C_{i 4}$, where $C_{i 1}$ is the number of MBFs in a block, $C_{i 2}-$ is the number of isomorphic blocks, $C_{i 4}-$ the number of similar nonisomorphic blocks, which contains the same number of MBFs per block and the same number of nonisomorphic blocks, $i$ is a type of block (total 4 ). The summation is over all the groups of blocks of each type with the same number of MBFs and with the same number of isomorphic blocks. But unlike formulas 1-4 the Dedekind number is calculated non-recursively, but for the MBFs of the same rank from where the blocks is taken.

For example, there are 2 nonisomorphic blocks of the second type for the third rank containing 1 disjunctive self-complementary MBF and 1 self-dual MBF. There are only 2 groups of blocks with the same number of MBFs and with the same number of isomorphic blocks. The first group consists of one block of 5 MBFs. The second one consists of three isomorphic blocks with $5 \mathrm{MBFs}$ in each. So, $D(3)=5 \cdot 1 \cdot 1+5 \cdot 3 \cdot 1=20$.

Let's graph groupoids with unity which graphs will correspond to the MBF blocks.

Let's draw a graph with six items for the first type MBF block.

Let's lay off generators $a$ and $b$, from starting element 1 (matches some MBF) and draw the graph. If the third generator $c=a b a$, which corresponds to the conjunctive complement in the original block, are entered into this graph then the groupoid graph will be obtained, which fully corresponds to the first type MBF block (Figure 3):

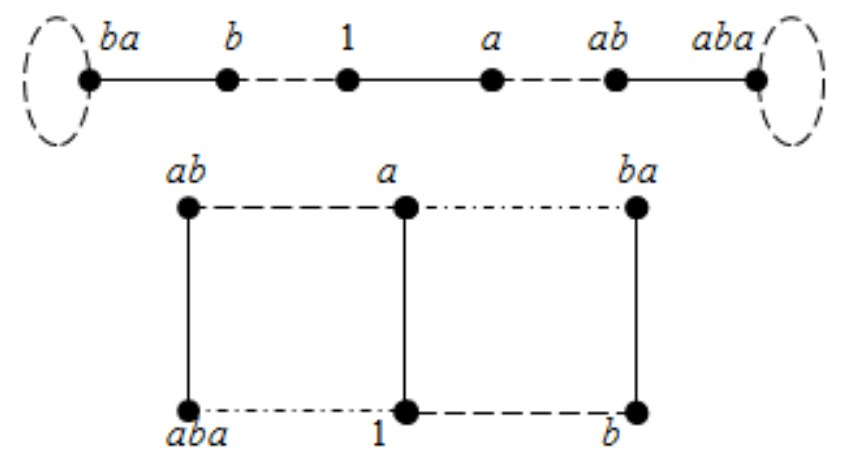

Figure 3. The groupoid graph to the first type MBF blocks.

Table 2. The Cayley table for the groupoid of the first type blocks.

\begin{tabular}{|c|c|c|c|c|c|c|}
\hline & $\boldsymbol{1}$ & $\boldsymbol{a}$ & $\boldsymbol{b}$ & $\boldsymbol{a b}$ & $\boldsymbol{b a}$ & $\boldsymbol{a b a}$ \\
\hline $\boldsymbol{1}$ & 1 & $a$ & $b$ & $a b$ & $b a$ & $a b a$ \\
\hline $\boldsymbol{a}$ & $a$ & 1 & $a b$ & $b$ & $a b a$ & $b a$ \\
\hline $\boldsymbol{b}$ & $b$ & $b a$ & 1 & $b a$ & $a$ & $b$ \\
\hline $\boldsymbol{a b}$ & $a b$ & $a b a$ & $a$ & $a b a$ & 1 & $a b$ \\
\hline $\boldsymbol{b a}$ & $b a$ & $b$ & $b a$ & 1 & $b$ & $a$ \\
\hline $\boldsymbol{a b a}$ & $a b a$ & $a b$ & $a b a$ & $a$ & $a b$ & 1 \\
\hline
\end{tabular}

As far as $(a b \cdot a b) a b=a$ and $a b(a b \cdot a b)=a b$, the operation is not associative and this groupoid is not a semigroup. In addition, the operation in the table is not always decidable from the right: $\nexists x:(a b \cdot x=b)$, i.e. the groupoid is not a quasigroup, too. However, the groupoid operation is always decidable from the left, because for any elements $\alpha$ and $\beta \exists y:(y \cdot \alpha=\beta)$. This follows from the table, as all the elements in the columns are different, but not in lines.

Let's develop defining relations for this groupoid:

$$
a^{2}=1, b^{2}=1, a b a b=a b a, b a b=b a
$$

Let us set $s=\left[\frac{n}{4}\right]$ and develop defining relations for of the first type blocks. It's possible both $n \equiv 0(\bmod 4)$ and $n \equiv 2(\bmod 4)$. If $n \equiv 0(\bmod 4)$, then:

$$
a^{2}=1, b^{2}=1,(a b)^{s-1} a=(a b)^{s},(b a)^{s}=(b a)^{s} b,
$$

The operation in this groupoid is not associative due to

$\left((a b)^{s} \cdot(a b)^{s}\right) \cdot(a b)^{s}=(b a)^{s} ;(a b)^{s} \cdot\left((a b)^{s} \cdot(a b)^{s}\right)=(a b)^{s}$

where $(a b)^{s} \cdot(a b)^{s}=b$.

If $n \equiv 2(\bmod 4)$ and $n \neq 2$, then:

$$
a^{2}=1, b^{2}=1,(a b)^{s+1}=(a b)^{s} a,(b a)^{s} b=(b a)^{s}
$$

In this case the operation is not associative, too, due to

$$
\begin{aligned}
& \left((a b)^{s} \cdot(a b)^{s}\right) \cdot(a b)^{s}=a(a b)^{s-1} ; \\
& (a b)^{s} \cdot\left((a b)^{s} \cdot(a b)^{s}\right)=(a b)^{s}
\end{aligned}
$$

where $(a b)^{s} \cdot(a b)^{s}=a b a$.

Our groupoid degenerates into an Abelian group, namely, into the second-order cyclic group $C_{2}$ due to the defining relation reduces to $a^{2}=1 ; b=1$ for $n=2$.

Let's graph a groupoid for the second type blocks containing five elements. By introducing the third generator $c=a b a$, we will obtain an MBF block ICF containing five elements (Figure 4):

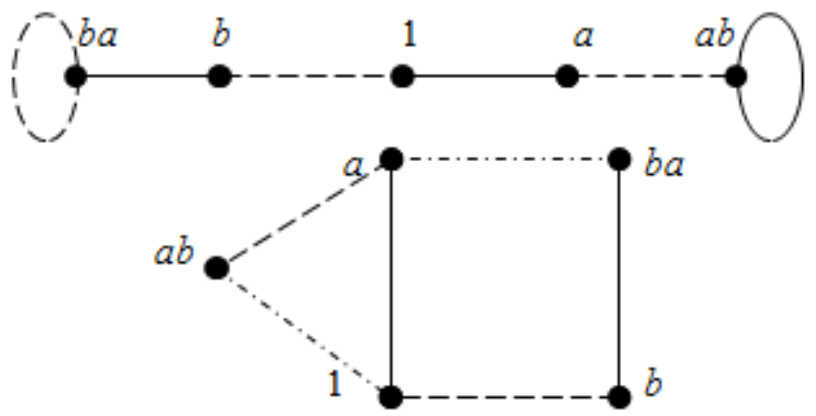

Figure 4. The groupoid graph to the second type MBF blocks. 
Table. 3. The Cayley table for the groupoid of the second type blocks.

\begin{tabular}{|c|c|c|c|c|c|}
\hline & $\boldsymbol{1}$ & $\boldsymbol{a}$ & $\boldsymbol{b}$ & $\boldsymbol{a b}$ & $\boldsymbol{b a}$ \\
\hline $\boldsymbol{1}$ & 1 & $a$ & $b$ & $a b$ & $b a$ \\
\hline $\boldsymbol{a}$ & $a$ & 1 & $a b$ & $b$ & $a b$ \\
\hline $\boldsymbol{b}$ & $b$ & $b a$ & 1 & $b a$ & $a$ \\
\hline $\boldsymbol{a} \boldsymbol{b}$ & $a b$ & $a b$ & $a$ & $a$ & 1 \\
\hline $\boldsymbol{b a}$ & $b a$ & $b$ & $b a$ & 1 & $b$ \\
\hline
\end{tabular}

The following defining relations can be obtained from the table:

$$
a^{2}=1, b^{2}=1, a b a=a b, b a b=b a
$$

If $(a b \cdot a b) a b=b$ and $a b(a b \cdot a b)=a b$, the operation is not associative. So, we obtain a groupoid with two generators $a$ and $b$ and unity c.

There generally are two cases $n \equiv 1(\bmod 4)$ and $n \equiv 3(\bmod 4)$ for the second type block. If $n \equiv 1(\bmod 4)$ the defining relations are as follows:

$$
a^{2}=1, b^{2}=1,(a b)^{s}=(a b)^{s} a,(b a)^{s}=(b a)^{s} b
$$

The operation is not associative due to

$$
\left((a b)^{s} \cdot(a b)^{s}\right) \cdot(a b)^{s}=(b a)^{s-1} b ;(a b)^{s} \cdot\left((a b)^{s} \cdot(a b)^{s}\right)=(a b)^{s}
$$

where $(a b)^{s} \cdot(a b)^{s}=a$.

If $n \equiv 3(\bmod 4)$ and $n \neq 3$ the defining relations are as follows:

$$
a^{2}=1, b^{2}=1,(a b)^{s} a=(a b)^{s+1},(b a)^{s} b=(b a)^{s+1}
$$

The operation is not associative in this case, too, due to $\left((a b)^{s} \cdot(a b)^{s}\right) \cdot(a b)^{s}=a(a b)^{s-1} ;(a b)^{s} \cdot\left((a b)^{s} \cdot(a b)^{s}\right)=(a b)^{s}$ where $(a b)^{s} \cdot(a b)^{s}=a b a$.

$$
(a \cdot b) a=1 \text { and } a(b \cdot a)=a \text { is true if } n=3
$$

The groupoid for the third type blocks is the same as for the thirst one except for generators $a$ and $b$ which interplace in all the expressions (Figure 5):

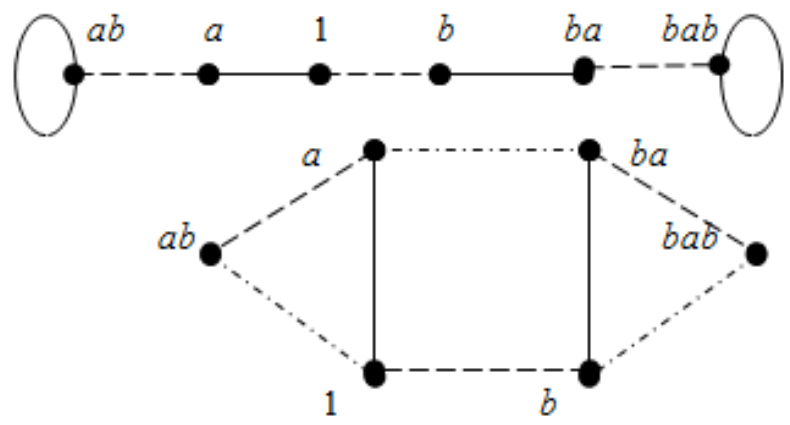

Figure 5. The groupoid graph to the third type MBF blocks.
There are two cases, too: $n \equiv 0(\bmod 4)$ and $n \equiv 2(\bmod 4)$. If $n \equiv 0(\bmod 4)$, then:

$$
a^{2}=1, b^{2}=1,(b a)^{s-1} b=(b a)^{s},(a b)^{s}=(a b)^{s} a
$$

The operation is not associative due to

$$
\begin{aligned}
& \left((b a)^{s} \cdot(b a)^{s}\right) \cdot(b a)^{s}=(a b)^{s} \\
& (b a)^{s} \cdot\left((b a)^{s} \cdot(b a)^{s}\right)=(b a)^{s}
\end{aligned}
$$

It is the same if $n \equiv 2(\bmod 4)$ and $n \neq 2$ :

$$
a^{2}=1, b^{2}=1,(b a)^{s+1}=(b a)^{s} b,(a b)^{s} a=(a b)^{s}
$$

The operation is not associative due to

$$
\begin{aligned}
& \left((b a)^{s} \cdot(b a)^{s}\right) \cdot(b a)^{s}=b(b a)^{s-1} \\
& (b a)^{s} \cdot\left((b a)^{s} \cdot(b a)^{s}\right)=(b a)^{s}
\end{aligned}
$$

The groupoid degenerates the second-order cyclic group $C_{2}$ due to the defining relation reduces to $a^{2}=1 ; b=1$ for $n=2$.

The groupoid is as follows for the fourth type cyclic MBF blocks (example, for six elements) (Figure 6):
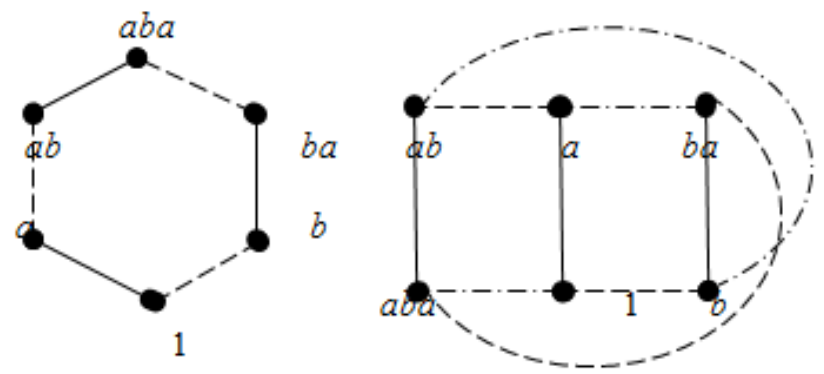

Figure 6. The groupoid graph to the fourth type MBF blocks.

Table 4. The Cayley table for the groupoid of the fourth type blocks.

\begin{tabular}{|c|c|c|c|c|c|c|}
\hline$\cdot$ & $\mathbf{1}$ & $\boldsymbol{a}$ & $\boldsymbol{b}$ & $\boldsymbol{a b}$ & $\boldsymbol{b a}$ & $\boldsymbol{a b a}$ \\
\hline $\mathbf{1}$ & 1 & $a$ & $b$ & $a b$ & $b a$ & $a b a$ \\
\hline $\boldsymbol{a}$ & $a$ & 1 & $a b$ & $b$ & $a b a$ & $b a$ \\
\hline $\boldsymbol{b}$ & $b$ & $b a$ & 1 & $a b a$ & $a$ & $a b$ \\
\hline $\boldsymbol{a b}$ & $a b$ & $a b a$ & $a$ & $b a$ & 1 & $b$ \\
\hline $\boldsymbol{b a}$ & $b a$ & $b$ & $a b a$ & 1 & $a b$ & $a$ \\
\hline $\boldsymbol{a b a}$ & $a b a$ & $a b$ & $b a$ & $a$ & $b$ & 1 \\
\hline
\end{tabular}

The defining relations for this groupoid are $a^{2}=1, b^{2}=1,(a b)^{3}=1$

Here the operation is associative but noncommutative. We have dihedral group $D_{3}$ in this case.

There generally are the following defining relations for the fourth type blocks:

$$
a^{2}=1, b^{2}=1,(a b)^{2 s}=1
$$


We have dihedral group $D_{n / 2}$ in this case and the operation is noncommutative. Except for the fourth-order group, the Klein group or $D_{2}$ which will be commutative.

Note that $n$ is always even for the first, the third and the fourth type blocks and it is always odd for the second ones.

\section{REFERENCES}

[1] Dedekind R. Uber Zerlegungen von Zahlen durch ihre grössten gemainsamen Teilor // Festschrift Hoch. Braunschweig u. ges. Werke. II. — 1897. — S. 103-148.

[2] Church, Randolph Numerical analysis of certain free distributive structures. Duke Math. J. 6 (1940). 732-734.

[3] Ward, Morgan Note on the order of free distributive lattices. Bull. Amer. Math. Soc. 52 (1946), 423-432
[4] Church, Randolph (1965), "Enumeration by rank of the free distributive lattice with 7 generators", Notices Amer. Math. Soc. 11: 724 .

[5] Wiedemann, Doug A computation of the eighth Dedekind number. Order 8 (1991), no. 1, 5-6.

[6] T.Stephen, T.Yusun Counting inequivalent Monotone Boolean Functions. arXiv: 1209.4623v1 [cs.DS] 20 Sep 2012.

[7] Pieter-Jan Hoedt. Parallelizing with MPI in Java to Find the ninth Dedekind Number.

http://people.cs.kuleuven.be/ bart.demoen/WVKULAK/first draftofPaper_Hoedt.pdf.

[8] Иваницкий А.М., Ткаченко В.Г. Взаимосвязь между матроидами и монотонными булевыми функциями электрических цепей. Наукові праці ОНАЗ ім. О.С. Попова. 2009. № 1. С. 18 - 26.

[9] Tkachenco V.G., Sinyavsky O.V. (2017). Basic MBF Blocks Properties and Rank 6 Blocks. Computer Science and Information Technology 5(1): pp.27-36, 2017 DOI: 10.13189/csit.2017.050104. 